https://doi.org/10.22319/rmcp.v12i4.5016

Nota de investigación

\title{
Comparación de ecuaciones para ajustar curvas de crecimiento de vacas Holstein, Jersey y Jersey $x$ Holstein en pastoreo
}

\author{
Sonia Contreras Piña ${ }^{a}$ \\ José Guadalupe García Muñiz ${ }^{\text {a* }}$ \\ Rodolfo Ramírez Valverde ${ }^{a}$ \\ Rafael Núñez Domínguez ${ }^{\mathrm{a}}$ \\ Citlalli Celeste González Ariceaga ${ }^{a}$
}

a Universidad Autónoma Chapingo. Departamento de Zootecnia, Posgrado en Producción Animal, km 38.5 Carretera México-Texcoco, 56230, Chapingo, Estado de México, México.

*Autor de correspondencia: jgarciamppa@hotmail.com

\section{Resumen:}

El objetivo del estudio fue comparar la bondad de ajuste de cuatro ecuaciones no lineales para describir las curvas de crecimiento de vacas Jersey, Holstein, y Jersey x Holstein en pastoreo. Las ecuaciones de Brody, Gompertz, von Bertalanffy, y Logística se ajustaron a los datos $(n=2,315)$ de peso y edad de vacas Jersey $(n=54)$, Holstein $(n=6)$ y Jersey x Holstein $(\mathrm{n}=30)$. Para cada animal, genotipo y ecuación, se estimaron los parámetros $A, b$ y $k$ que produjeron las curvas de crecimiento de mejor ajuste. En cada una de las cuatro ecuaciones comparadas, el parámetro $A$ corresponde a la asíntota superior de la curva y estima el 'peso maduro' del animal, los parámetros $b$ y $k$ representan la constante de integración y la tasa de maduración. Para las curvas de crecimiento de las vacas Jersey y las cruzas de Jersey x Holstein, las ecuaciones de Gompertz y de von Bertalanffy produjeron el mejor ajuste. En contraste, la ecuación Logística tuvo el mejor ajuste para las curvas de crecimiento de Holstein, seguida muy de cerca por las ecuaciones de Gompertz y von Bertalanffy. En las condiciones de manejo y alimentación de los animales en este estudio, las curvas de 
crecimiento de las hembras de los tres genotipos estudiados pueden ajustarse con la ecuación de von Bertalanffy.

Palabras clave: Ganado lechero; modelos no lineales; patrón de crecimiento.

Recibido: $15 / 08 / 2018$

Aceptado: 17/02/2021

Cuando se tiene el peso de un mismo individuo a intervalos regulares de edad, desde el nacimiento hasta la madurez, es posible ajustar una función que describa la curva de peso de acuerdo con la edad. A esta curva que permite condensar la trayectoria de crecimiento de un individuo en unos cuantos parámetros se le denomina curva de crecimiento ${ }^{(1)}$.

Existen diversas ecuaciones que se han utilizado para describir el crecimiento de plantas y animales $^{(2)}$, entre ellas la de Brody ${ }^{(3)}$, Gompertz $^{(4)}$, Logística ${ }^{(5)}$ y von Bertalanffy ${ }^{(5)}$, todas casos especiales de la ecuación de Richards ${ }^{(5)}$. Las curvas de crecimiento reflejan las interrelaciones entre el impulso inherente del individuo de crecer y madurar, y el ambiente en el que se expresan estos impulsos ${ }^{(6)}$. Algunas de estas ecuaciones se han utilizado para describir el crecimiento de varias especies de interés zootécnico, como bovinos Pantaneira en $\operatorname{Brasil}^{(7)}$, y de hembras Holstein para predecir su crecimiento desde el nacimiento hasta el primer parto ${ }^{(8)}$. Estas ecuaciones también se han utilizado para describir el crecimiento de genotipos ovinos $^{(9,10,11)}$, bovinos productores de leche ${ }^{(12,13)}$, y caballos ${ }^{(14)}$.

En sistemas de producción de leche en pastoreo con bajo uso de alimento suplementario y una mezcla de genotipos bovinos que difieren en tamaño maduro, el peso promedio de la vaca determina en gran medida la máxima carga animal que soporta el sistema. Diversos genotipos bovinos pueden presentar diferentes pesos maduros y requerimientos de energía metabolizable para mantenimiento. El ajuste de una curva de crecimiento desde el nacimiento hasta la madurez puede ser útil para calcular los requerimientos de mantenimiento durante la vida del animal. Las curvas de crecimiento de animales individuales pueden estimarse ajustando modelos mixtos no lineales, aun con registros incompletos de la curva de crecimiento de los animales del hato. El objetivo de este estudio fue comparar la bondad de ajuste de cuatro ecuaciones no lineales, para describir las curvas de crecimiento de vacas Jersey, Holstein, y Jersey x Holstein en pastoreo de praderas mixtas de alfalfa-ballico perenne-ovillo-trébol blanco.

El estudio se realizó en el Módulo de Producción de Leche Orgánica en Pastoreo de la Granja Experimental de la Universidad Autónoma Chapingo, México. Díaz ${ }^{(15)}$ menciona 
detalladamente el desarrollo del Módulo: brevemente, el módulo se inició con 10 vacas Holstein y 25 vacas Jersey de primer parto en mayo de 2000. Los animales que integran el hato son vacas Jersey de la línea genética de Nueva Zelanda, vacas Holstein de la línea genética de Norteamérica y vacas originadas de la cruza de vacas Holstein con semen Jersey.

La alimentación de los animales se basa en el pastoreo de las praderas, heno que de éstas se conserva y ensilado de maíz que se produce en áreas de pradera que se renuevan cada año. Los animales se manejan en pastoreo intensivo rotacional en franjas, de praderas mixtas de alfalfa (Medicago saltiva) variedad Aragonesa, pasto ovillo (Dactylis glomerata) variedad Potomac, pasto ballico perenne (Lolium perenne) variedad Linn y trébol blanco (Trifolium repens) variedad Ladino.

Los animales del hato se manejan en tres grupos: lactantes, secas y animales en crecimiento. Dependiendo de las condiciones de crecimiento de la pradera se asignan potreros por grupo o se realiza el pastoreo con el esquema de líderes y seguidores, los líderes son las vacas en producción y los seguidores el grupo de vacas secas y ganado en crecimiento.

Con este esquema de pastoreo se provee a los animales de condiciones de producción que garantizan suficiente libertad de movimiento, área para descanso, aire fresco, luz y temperatura adecuada, agua limpia y alimento suficiente para la etapa productiva del animal. Además del forraje de la pradera, a los animales se le proporciona una mezcla mineral a libre acceso. Dicha mezcla fue formulada con base en un diagnóstico del estado mineral de los animales y del forraje de la pradera, con análisis en sangre, forraje, heces, pelo y leche ${ }^{(16)}$.

Las vacas paren en la pradera, en el grupo de vacas secas, proporcionándoles asistencia sólo cuando es necesario. La cría permanece con la madre en la pradera durante los primeros dos días de vida consumiendo calostro a libertad. Al tercer día, la madre se integra al grupo de vacas lactantes, la cría se envía a una sala de crianza con ventilación natural y se le asigna una jaula individual con piso elevado. Solo para las hembras, se abre una tarjeta en la cual se registra identificación de la cría, fecha y peso al nacimiento, identificación de la madre y del padre. La identificación de la cría consiste en tatuaje en la oreja derecha y arete en la oreja izquierda, usando un sistema de cuatro dígitos. Durante las dos primeras semanas de vida, las crías reciben cuatro litros de leche entera repartidos en dos tomas. Posteriormente, además de la leche las crías tienen acceso al forraje de la pradera. El destete se realiza a los tres meses de $\operatorname{edad}^{(15)}$.

Los registros de peso y edad de los animales de los genotipos estudiados se generaron a partir de las fechas de nacimiento, fechas de pesada y registros del peso vivo de animales individuales en diferentes etapas de su vida, desde el nacimiento hasta la madurez. Los animales se pesaron periódicamente en intervalos regulares de aproximadamente $30 \mathrm{~d}$. Se utilizó una báscula digital Tru-Test (Tru-Test, Palmerston North, NZ), Sistema EC 2000 con 
Barras GP 600, con capacidad de $1,500 \mathrm{~kg}$ y precisión de $0.5 \mathrm{~kg}$. Los datos de peso vivo utilizados en este estudio se registraron de 2012 a 2016. Los animales incluidos en el estudio se pesaron al nacimiento, al destete y mensualmente.

En total se contó con 2,315 registros de peso $(\mathrm{kg})$ y edad (d) generados por 54 vacas Jersey, 6 Holstein, y 30 Jersey x Holstein, con edades que fluctuaron de 1 a 6,597 d.

Los modelos de Brody ${ }^{(3)}$, una versión modificada de la función de Gompertz ${ }^{(4)}$, Logístico ${ }^{(5)}$ y de von Bertalanffy ${ }^{(5)}$, se ajustaron a los datos de peso y edad de las vacas.

Brody: $Y_{t}=A\left(1-b e^{-k t}\right)$

Gompertz: $Y_{t}=A e^{-e^{(b-k t)}}$

Logístico: $Y_{t}=A\left(1+b e^{-k t}\right)^{-1}$

von Bertalanffy: $Y_{t}=A\left(1-b e^{-k t}\right)^{3}$

En todos los casos, $\mathbf{Y}_{\mathrm{t}}$ es el peso vivo (kg) registrado a la edad $t$ (días); el parámetro $\boldsymbol{A}$ corresponde a la asíntota superior, que estima el peso $(\mathrm{kg})$ maduro del animal; el parámetro $\boldsymbol{b}$ es una constante de integración relacionada con el peso inicial; el parámetro $\boldsymbol{k}$ es la tasa de maduración; y $\boldsymbol{e}$ es la base de los logaritmos naturales ${ }^{(12)}$.

Con la información de peso y edad se calcularon los estadísticos descriptivos en diferentes secciones de la curva de crecimiento del hato. Se generaron nueve intervalos de edad: tres que cubrieron el intervalo del nacimiento hasta un año, y seis que cubrieron el intervalo de dos a doce años, con dos años de separación entre ellos. Una partición similar se hizo para describir la curva de crecimiento de ganado Angus de Norteamérica ${ }^{(17)}$. Los estadísticos descriptivos se obtuvieron utilizando el procedimiento MEANS de SAS $^{(18)}$.

Para cada genotipo se ajustaron cada una de las ecuaciones evaluadas, mediante el procedimiento NLMIXED de $\operatorname{SAS}^{(18)}$. Sólo el parámetro relacionado con el peso maduro $(\boldsymbol{A})$ se ajustó como efecto aleatorio en la ecuación respectiva; los restantes parámetros de la curva de crecimiento ( $\boldsymbol{b}$ y $\boldsymbol{k}$ ) se consideraron fijos. Se siguió un procedimiento similar al de otros estudios en los que se ajustaron modelos estocásticos para describir la variación individual en el crecimiento de animales de granja ${ }^{(19,20,21)}$. La inclusión de un término aleatorio $\left(a_{i}\right)$ asociado con el parámetro $\boldsymbol{A}$ de las ecuaciones de crecimiento comparadas, se hizo como lo

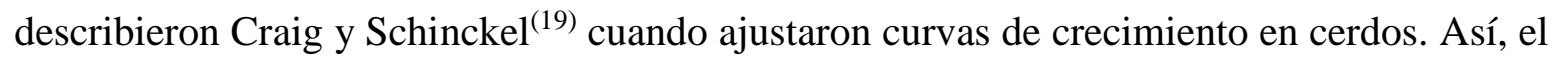
modelo estadístico ajustado dentro de genotipo, para cada una de las ecuaciones comparadas en el presente estudio, incluyó un término aleatorio relacionado con el tamaño maduro de la vaca, se expresa de la siguiente manera: 
$\begin{array}{lll}\text { Brody: } & Y_{i, t}=\left(A+a_{i}\right)\left(1-b e^{-k t}\right)+\varepsilon_{i, t} \\ \text { Gompertz: } & Y_{i, t}=\left(A+a_{i}\right) e^{-e^{(b-k t)}+\varepsilon_{i, t}} \\ \text { Logístico: } & Y_{i, t}=\left(A+a_{i}\right)\left(1+b e^{-k t}\right)^{-1}+\varepsilon_{i, t} \\ \text { von Bertalanffy: } & Y_{i, t}=\left(A+a_{i}\right)\left(1-b e^{-k t}\right)^{3}+\varepsilon_{i, t}\end{array}$

donde $\mathrm{Y}_{i, t}$ es el peso vivo del animal $i$ registrado en el día $t$ de edad, $e$ es la base de los logaritmos naturales (i.e. 2.718281), $\boldsymbol{A}$ es el peso maduro predicho, $a_{i}$ es el efecto aleatorio del animal $i$ para el parámetro de la curva de crecimiento relacionado con el peso maduro $(\boldsymbol{A})$ del animal $\sim \operatorname{Normal}\left(0, \sigma_{A}^{2}\right)$, $t$ es la edad del animal en días, $b$ es una constante de integración relacionada con el peso inicial del animal, $k$ es la tasa de maduración y $\varepsilon_{\mathrm{i}, \mathrm{t}}$ es el residual del modelo $\sim \operatorname{Normal}\left(0, \sigma_{\varepsilon}^{2}\right)$.

El ajuste de estos modelos generó, además de los estimadores de los parámetros de la curva de crecimiento, estimadores de varianza para el parámetro $\boldsymbol{A}\left(\sigma_{A}^{2}\right)$ y de la varianza residual $\left(\sigma_{\varepsilon}^{2}\right)$. El ajuste del modelo también generó estimadores de $-2 \log$ likelihood, el Criterio de Información de Akaike (AIC) y el Criterio de Información Bayesiano (BIC), que se utilizaron como criterios de bondad de ajuste para comparar las cuatro ecuaciones ajustadas.

El ajuste de cada uno de los modelos a los tres genotipos estudiados generó los coeficientes de los parámetros $\boldsymbol{A}, \boldsymbol{b}$ y $\boldsymbol{k}$, para producir las curvas de crecimiento de animales individuales. En el código SAS utilizado, también se incluyó una expresión para generar los coeficientes de la regresión fija para describir la curva de crecimiento promedio de cada genotipo estudiado. Estos coeficientes se utilizaron para producir, en cada ecuación y genotipo, los valores predichos de peso vivo para generar las curvas de animales individuales y las curvas de crecimiento promedio por genotipo de la vaca, utilizando el procedimiento SGPLOT de $\mathrm{SAS}^{(18)}$.

Los estadísticos descriptivos del peso vivo para distintos intervalos de edad de la curva de crecimiento de los genotipos evaluados se presentan en el Cuadro 1. En general, se observa que el peso se incrementa notablemente en los primeros tres a cuatro años, posteriormente se mantiene relativamente constante. La desviación estándar se incrementa conforme avanza la edad del animal; esto se explica por una mayor variación en el peso adulto en los diferentes genotipos $^{(22)}$. 
Cuadro 1: Estadísticos descriptivos de peso $(\mathrm{kg})$ agrupado por edad de hembras Jersey, Holstein y Jersey $\mathrm{x}$ Holstein manejadas en pastoreo

\begin{tabular}{lllrrrrrrr}
\hline \multirow{2}{*}{ Edad (días) } & \multicolumn{3}{c}{ Jersey } & \multicolumn{4}{c}{ Holstein } & \multicolumn{3}{c}{ Jersey x Holstein } \\
\cline { 2 - 10 } & $\mathbf{n}$ & Media & \multicolumn{1}{c}{ DE } & \multicolumn{1}{c}{$\mathbf{n}$} & Media & DE & n & Media & \multicolumn{1}{c}{ DE } \\
\hline $0-30$ & 10 & 26.2 & 6.6 & -- & -- & -- & 7 & 26.0 & 3.4 \\
$30-120$ & 19 & 56.1 & 11.9 & -- & -- & -- & 2 & 69.5 & 21.9 \\
$121-200$ & 44 & 81.6 & 14.2 & 2 & 110.5 & 2.1 & 27 & 104.2 & 34.4 \\
$201-365$ & 92 & 128.4 & 29.5 & 5 & 136.2 & 24.9 & 72 & 151.4 & 35.1 \\
$366-730$ & 201 & 245.9 & 46.3 & 12 & 276.0 & 56.6 & 157 & 255.3 & 58.9 \\
$731-1460$ & 404 & 338.5 & 54.3 & 28 & 490.5 & 66.5 & 269 & 365.0 & 60.4 \\
$1461-2190$ & 213 & 358.5 & 51.1 & 43 & 477.0 & 42.5 & 138 & 441.7 & 56.3 \\
$2191-2920$ & 103 & 384.4 & 33.4 & 55 & 512.0 & 37.0 & 23 & 480.7 & 55.1 \\
$>2920$ & 235 & 403.0 & 58.0 & 130 & 527.2 & 44.5 & 11 & 464.0 & 28.9 \\
\hline
\end{tabular}

$\mathrm{DE}=$ desviación estándar.

Los estimadores de bondad de ajuste del modelo, $-2 \log$ likelihood, el Criterio de Información de Akaike (AIC) y el Criterio de Información Bayesiano (BIC), se presentan en el Cuadro 2. De acuerdo con la magnitud de estos estimadores (valores más pequeños indican mejor ajuste), los modelos de von Bertalanffy y de Gompertz fueron los que mejor ajustaron las curvas de crecimiento de hembras del genotipo Jersey. Para las curvas de crecimiento de las cruzas Jersey x Holstein el mejor ajuste se obtuvo con la ecuación de von Bertalanffy, y para Holstein con la Logística, seguida muy de cerca por las ecuaciones de Gompertz y von Bertalanffy.

Cuadro 2: Valores de los criterios utilizados para comparar el ajuste de las ecuaciones a los registros de peso y edad de bovinos de tres genotipos

\begin{tabular}{clccc}
\hline \multirow{2}{*}{ Genotipo } & & \multicolumn{3}{c}{ Indicador de bondad de ajuste del modelo } \\
\cline { 2 - 4 } & Modelo & $\mathbf{- 2}$ log likelihood & AIC & BIC \\
\hline \multirow{3}{*}{ Jersey (J) } & 12963 & 12973 & 12983 \\
& Brody & 12892 & 12902 & 12912 \\
& von Bertalanffy & 12891 & 12901 & 12911 \\
& Gompertz & 12920 & 12930 & 12940 \\
& Logístico & 2945 & 2955 & 2954 \\
& Brody & 2915 & 2925 & 2924 \\
Holstein (H) & von Bertalanffy & 2909 & 2919 & 2918 \\
& Gompertz & 2895 & 2905 & 2904 \\
& Logístico & 7143 & 7153 & 7260 \\
& Brody & 7111 & 7121 & 7128 \\
& von Bertalanffy & 7122 & 7132 & 7139 \\
& Gompertz & 7151 & 7161 & 7168 \\
\hline
\end{tabular}

${ }^{1}$ AIC y BIC son los Criterio de Información de Akaike (AIC) y Bayesiano (BIC) para evaluar la bondad de ajuste del modelo respectivo (valores más bajos indican mejor ajuste del modelo). 
En un estudio de crecimiento de bovinos en Brasil, la ecuación de Brody produjo el mejor ajuste para describir la curva de crecimiento de bovinos Nellore en confinamiento ${ }^{(23)}$. Igualmente, la ecuación de Brody ajustó mejor las curvas de crecimiento de bovinos Tropicarne, lo que implica para este genotipo una tasa de maduración lenta, característica del crecimiento de los bovinos en condiciones de pastoreo en el trópico ${ }^{(24)}$. Brown et al ${ }^{(25)}$ reportaron que la ecuación de Brody fue la que mejor ajustó la curva de crecimiento en varios genotipos bovinos bajo diferentes condiciones de manejo y alimentación. En contraste, otros investigadores $^{(26)}$ indican que las ecuaciones de Gompertz y de von Bertalanffy se ajustaron mejor para describir el crecimiento de novillos Hereford, lo que concuerda con lo encontrado en este estudio para los genotipos Jersey y Jersey x Holstein.

Los estimadores de los parámetros $\boldsymbol{A}, \boldsymbol{b}$ y $\boldsymbol{k}$ de los modelos de crecimiento evaluados, así como los estimadores de las varianzas del 'peso maduro' y residual se presentan en el Cuadro 3. El parámetro $\boldsymbol{A}$, que representa el peso maduro, fue diferente en cada genotipo, dependiendo de la ecuación utilizada. La ecuación de Brody ajustó para los tres genotipos el valor más alto del peso maduro, lo que concuerda con lo obtenido en otros estudios ${ }^{(23)}$, en los que también se menciona que esta ecuación sobreestima el peso maduro. Herrera et al ${ }^{(27)}$ indicaron que grupos de animales con mayor valor en $\boldsymbol{A}$ son menos precoces. Para Holstein, las ecuaciones de von Bertalanffy y Logística produjeron prácticamente el mismo valor de $\boldsymbol{A}$ $(512 \mathrm{~kg})$. Este valor del peso maduro para las vacas del genotipo Holstein está muy por debajo de los estimadores de 591, 566 y $543 \mathrm{~kg}$ obtenidos en Irlanda en condiciones de pastoreo para dos líneas de Holstein-Friesian de Norteamérica-Europa y Holstein-Friesian de Nueva Zelanda, respectivamente ${ }^{(13)}$. La ecuación de Brody produjo el valor más bajo de $\boldsymbol{k}$. Un valor pequeño de $\boldsymbol{k}$ representa una menor velocidad de crecimiento para llegar al peso asintótico a partir del peso inicial, o tasas de maduración más lentas ${ }^{(7,24,28)}$. 
Cuadro 3: Parámetros de la curva de crecimiento ( \pm error estándar), estimadores de las varianzas residual y del peso maduro después de ajustar las ecuaciones a los registros de peso y edad de bovinos de tres genotipos

\begin{tabular}{|c|c|c|c|}
\hline Parámetro & Jersey & Holstein & Jersey x Holstein \\
\hline \multicolumn{4}{|c|}{ Ecuación de Brody } \\
\hline$A$ & $400.0 \pm 6.5$ & $513.5 \pm 13.6$ & $455.8 \pm 9.4$ \\
\hline$b$ & $1.04 \pm 0.01$ & $0.99 \pm 0.03$ & $1.02 \pm 0.02$ \\
\hline$k$ & $1.71 \mathrm{E}^{-3} \pm 5.6 \mathrm{E}^{-5}$ & $1.95 \mathrm{E}^{-3} \pm 0.01$ & $1.65 \mathrm{E}^{-3} \pm 8.1 \mathrm{E}^{-5}$ \\
\hline$\sigma_{A}^{2}$ & $1005.0 \pm 156.8$ & $800.0 \pm 617.0$ & $803.0 \pm 121.0$ \\
\hline$\sigma_{e}^{2}$ & $1075.0 \pm 43.7$ & $1875.0 \pm 154.2$ & $1497.5 \pm 100.0$ \\
\hline \multicolumn{4}{|c|}{ Ecuación de Gompertz } \\
\hline$A$ & $383.5 \pm 5.6$ & $510.6 \pm 15.0$ & $426.6 \pm 7.9$ \\
\hline$b$ & $0.98 \pm 0.03$ & $1.30 \pm 0.01$ & $0.84 \pm 0.04$ \\
\hline$k$ & $3.19 \mathrm{E}^{-3} \pm 9.6 \mathrm{E}^{-5}$ & $3.86 \mathrm{E}^{-3} \pm 3.42 \mathrm{E}^{-4}$ & $2.97 \mathrm{E}^{-3} \pm 1.27 \mathrm{E}^{-4}$ \\
\hline$\sigma_{A}^{2}$ & $918.7 \pm 140.2$ & $904.8 \pm 2598.0$ & $952.4 \pm 143.2$ \\
\hline$\sigma_{e}^{2}$ & $996.4 \pm 39.7$ & $1731.2 \pm 148.4$ & $1238.0 \pm 68.0$ \\
\hline \multicolumn{4}{|c|}{ Ecuación Logística_ } \\
\hline$A$ & $373.5 \pm 5.3$ & $512.3 \pm 10.2$ & $413.8 \pm 7.5$ \\
\hline$b$ & $7.31 \pm 0.41$ & $13.2 \pm 2.86$ & $5.55 \pm 0.39$ \\
\hline$k$ & $4.69 \mathrm{E}^{-3} \pm 1.39 \mathrm{E}^{-4}$ & $5.43 \mathrm{E}^{-3} \pm 4.02 \mathrm{E}^{-4}$ & $4.26 \mathrm{E}^{-3} \pm 1.83 \mathrm{E}^{-4}$ \\
\hline$\sigma_{A}^{2}$ & $900.8 \pm 134.4$ & $500.6 \pm 437.7$ & $958.7 \pm 138.7$ \\
\hline$\sigma_{e}^{2}$ & $1000.4 \pm 39.3$ & $1661.5 \pm 141.8$ & $1282.7 \pm 70.6$ \\
\hline \multicolumn{4}{|c|}{ Ecuación de von Bertalanffy___ } \\
\hline$A$ & $388.4 \pm 5.9$ & $512.0 \pm 10.6$ & $432.3 \pm 8.6$ \\
\hline$b$ & $0.64 \pm 0.02$ & $0.92 \pm 0.16$ & $0.59 \pm 0.02$ \\
\hline$k$ & $2.70 \mathrm{E}^{-3} \pm 8.3 \mathrm{E}^{-5}$ & $3.45 \mathrm{E}^{-3} \pm 3.92 \mathrm{E}^{-4}$ & $2.57 \mathrm{E}^{-3} \pm 1.07 \mathrm{E}^{-4}$ \\
\hline$\sigma_{A}^{2}$ & $1013.7 \pm 163.9$ & $512.2 \pm 411.7$ & $1153.9 \pm 191.7$ \\
\hline$\sigma_{e}^{2}$ & $998.1 \pm 39.8$ & $1778.0 \pm 152.0$ & $1135.3 \pm 57.6$ \\
\hline
\end{tabular}

El ajuste de la ecuación de Brody a los datos de peso y edad de los tres genotipos estudiados se muestra en la Figura 1. En el presente trabajo fue reducida la disponibilidad de registros del nacimiento al destete al inicio del proyecto. Para los genotipos Jersey y Jersey x Holstein, la ecuación de Brody, en el caso del presente trabajo, predijo valores negativos para peso al nacimiento, mientras que para Holstein estimó pesos positivos pero bajos. Lo anterior concuerda con lo estimado por Mgbere y Olutogun ${ }^{(29)}$, quienes argumentan que la ecuación de Brody subestima el peso durante los primeros días de vida de ganado N'Dama, pero que ajusta bien a edades mayores que seis meses. Igualmente, Berry et $a l^{(13)}$ encontraron que la ecuación de Brody predice pesos negativos a edades tempranas cuando se utiliza para ajustar 
las curvas de crecimiento de vacas Holstein de las líneas genéticas de Norteamérica, Holstein Europea y Holstein-Friesian, manejadas en condiciones de semipastoreo. En Brasil ${ }^{(30)}$ encontraron que las ecuaciones de Brody y Gompertz tuvieron el mejor ajuste de la curva de crecimiento del nacimiento a la edad adulta de vacas Caracu. Lo anterior concuerda con el mejor ajuste de la ecuación de Brody para describir la curva de crecimiento de ganado de carne Lagune en condiciones de pastoreo ${ }^{(31)}$.

Figura 1: Diagrama de dispersión para pesos y edades del ajuste de las curvas de crecimiento generadas por la ecuación de Brody para hembras Jersey, Holstein y Jersey x Holstein

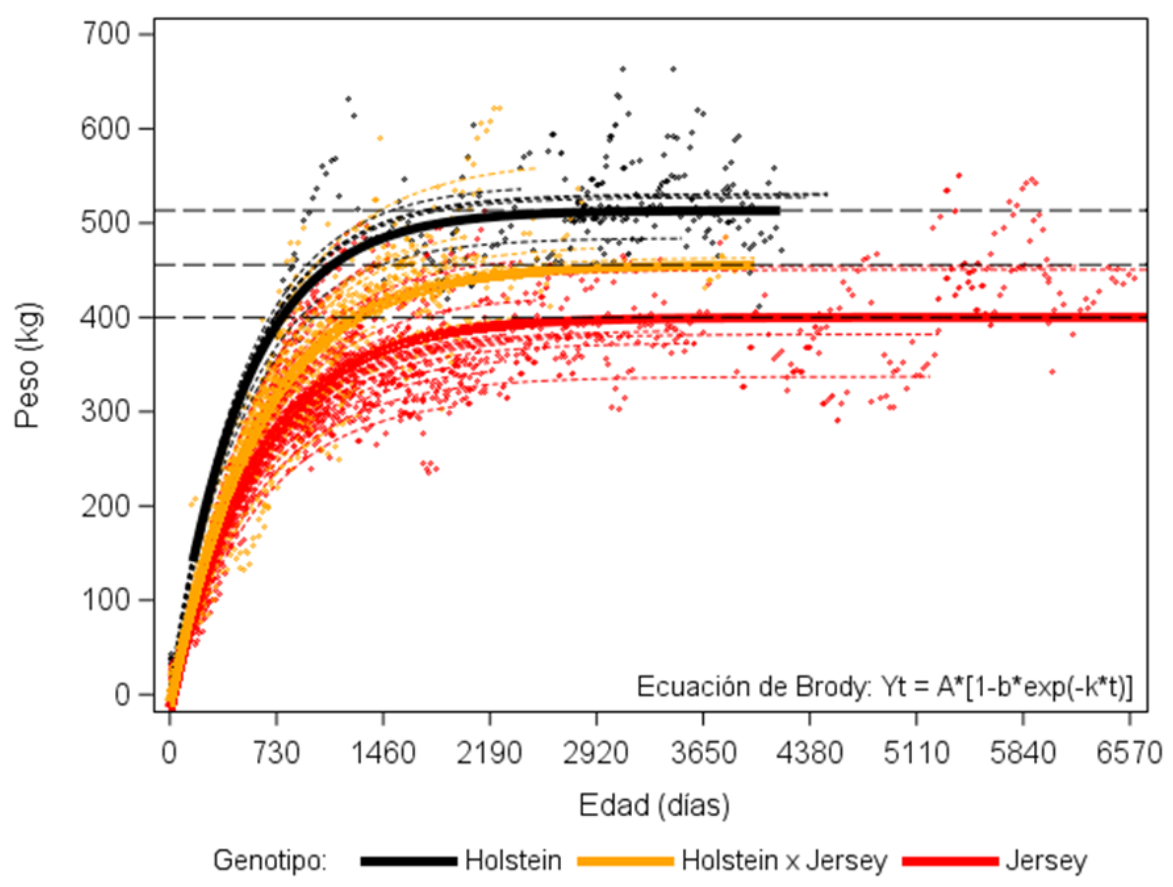

El ajuste de la ecuación de Gompertz a los datos de peso y edad de los tres genotipos estudiados se muestra en la Figura 2. En el presente estudio, los pesos al nacimiento para Holstein, predichos con el ajuste de la ecuación de Gompertz, fueron muy cercanos a cero, mientras que para Jersey y Jersey $\mathrm{x}$ Holstein se observó un mejor ajuste. Varios investigadores ${ }^{(32)}$ utilizaron esta ecuación para describir el crecimiento de bovinos lecheros, por ser la que mejor ajustó sus datos. Berry et al ${ }^{(13)}$ también encontraron que la ecuación de Gompertz ajustó mejor que la de Brody las curvas de crecimiento de vacas Holstein Americana, Holstein Europea y Holstein-Friesian, manejadas en condiciones de pastoreo. 
Figura 2: Diagrama de dispersión para pesos y edades del ajuste de las curvas de crecimiento generadas por la ecuación de Gompertz para hembras Jersey, Holstein y Jersey

$\mathrm{x}$ Holstein

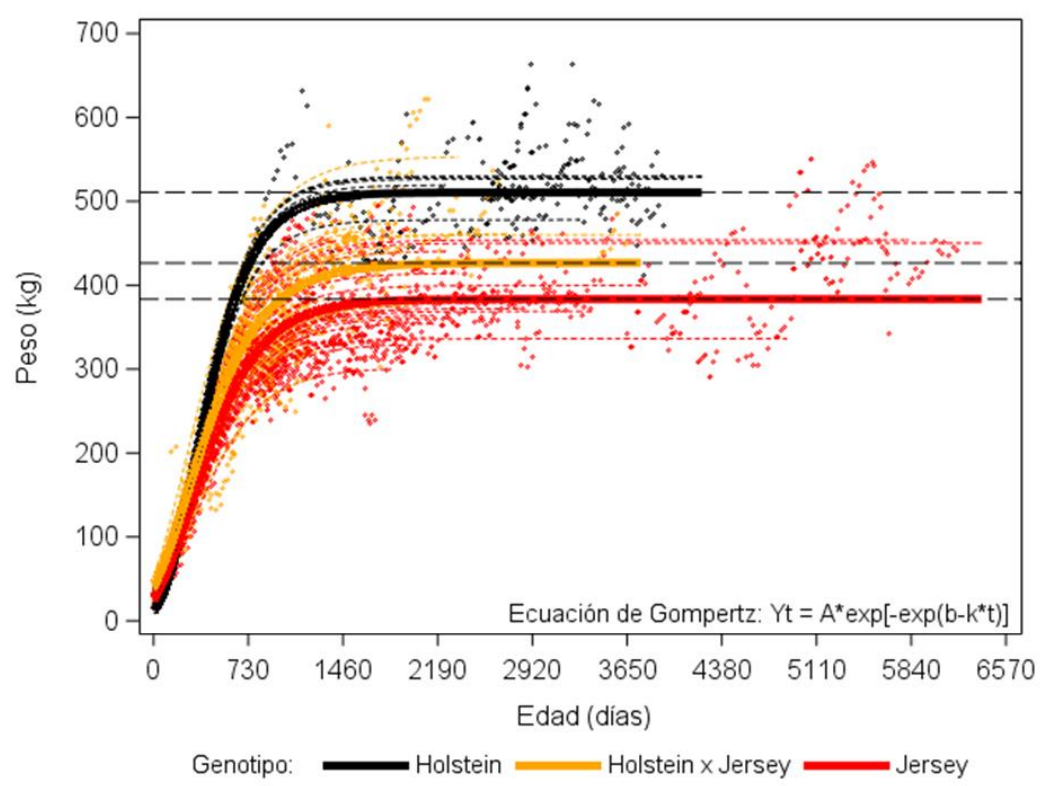

El ajuste de la ecuación Logística a los datos de peso y edad de los tres genotipos estudiados se muestra en la Figura 3. En estudios previos que describen la curva de crecimiento de ganado lechero, la ecuación Logística subestimó el peso maduro de los animales ${ }^{(12)}$. En este estudio, la ecuación Logística produjo valores idénticos a la de von Bertalanffy para este parámetro de la curva de crecimiento.

Figura 3: Diagrama de dispersión para pesos y edades del ajuste de las curvas de crecimiento generadas por la ecuación Logística para hembras Jersey, Holstein y Jersey x Holstein

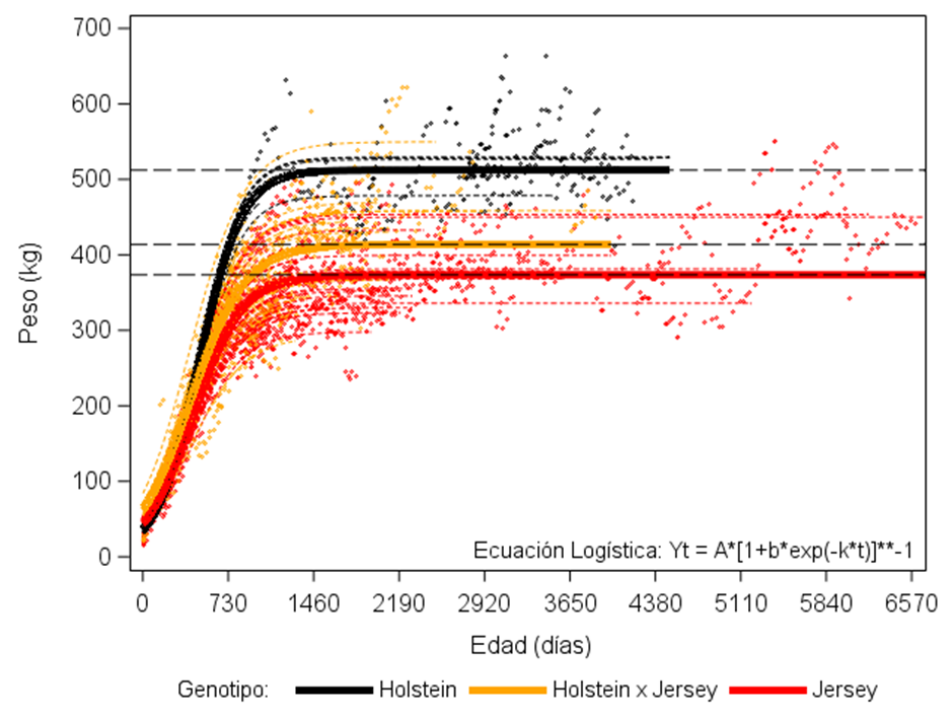


El ajuste de la ecuación de von Bertalanffy a los datos de peso y edad de los tres genotipos estudiados se muestra en la Figura 4. Esta ecuación ajustó los pesos vivos al nacimiento de Holstein con valores negativos y de Jersey y Jersey x Holstein, con pesos cercanos al cero, lo que difiere con lo reportado en otros estudios en los que la ecuación de von Bertalanffy sobreestimó el peso a edades tempranas en ganado $\mathrm{N}^{\prime} \mathrm{Dama}^{(29)}$. Investigadores españoles ${ }^{(33)}$ compararon la ecuación de Brody, Richards y von Bertalanffy para describir las curvas de crecimiento de vacas Retinta para carne en condiciones de pastoreo extensivo en España, encontrando que la ecuación de von Bertalanffy tuvo el mejor ajuste para el patrón de crecimiento de este genotipo. En el presente estudio, la ecuación de von Bertalanffy generó el mejor ajuste en las curvas de crecimiento de vacas Jersey y Jersey x Holstein, y para vacas Holstein produjo parámetros idénticos a los de la ecuación Logística, que produjo el mejor ajuste para las curvas de crecimiento de este genotipo.

Figura 4: Diagrama de dispersión para pesos y edades del ajuste de las curvas de crecimiento generadas por la ecuación de von Bertalanffy para hembras Jersey, Holstein y Jersey x Holstein

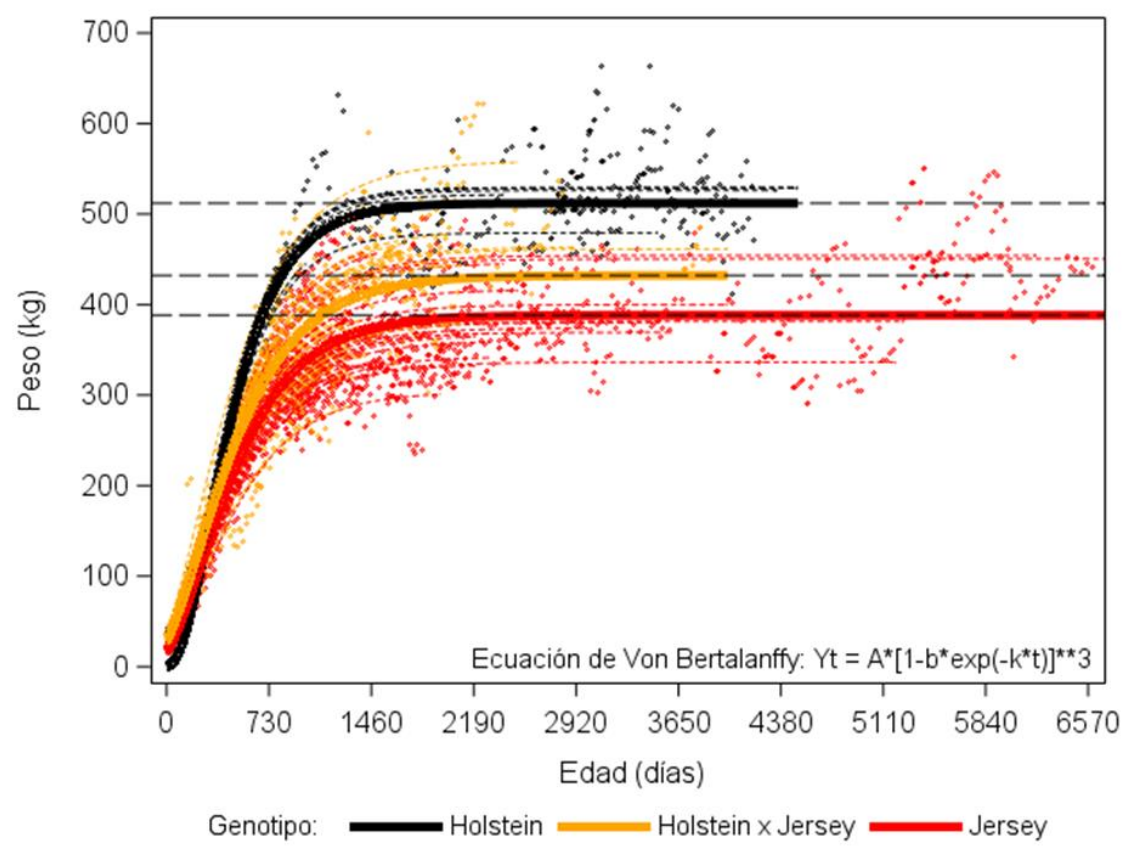

En conclusión, las ecuaciones de Gompertz y de von Bertalanffy describen mejor la curva de crecimiento de vacas Jersey; la de von Bertalanffy la de hembras Jersey x Holstein, y la Logística la de las hembras Holstein, seguida por las de Gompertz y von Bertalanffy. Para las condiciones de manejo y alimentación de los animales en este estudio, y con la finalidad de describir adecuadamente su patrón de crecimiento, las curvas de crecimiento de las hembras de los tres genotipos estudiados pueden ajustarse con la ecuación de von Bertalanffy. 


\section{Agradecimientos}

Al Consejo Nacional de Ciencia y Tecnología (CONACYT) por el apoyo económico otorgado al primer autor para realizar estudios de Maestría en Ciencias en la Universidad Autónoma Chapingo.

\section{Literatura citada:}

1.Val JE, Freitas MAR, Oliveira HN, Cardoso VL, Machado PF, Paneto JC. Indicadores de desempenho em rebanho da raça Holandesa: curvas de crescimento e altura, características reprodutivas, produtivas e parâmetros genéticos. Arq Bras Med Vet Zootec 2004;56(1):86-93.

2. Koya PR, Goshu AT. Solutions of rate-state equation describing biological growths. Am J Math and Stat 2004;3(6):305-311.

3. Thornley JHM, France J. Mathematical models in agriculture, 2nd ed. Wallingford: CABI Publishing; 2007.

4. Tjørve KMC, Tjørve E. The use of Gompertz models in growth analyses, and new Gompertz-model approach: an addition to the Unified-Richards family. PLoS One 2017;12(6) e0178691. https://doi.org/10.1371/journal.pone.0178691.

5. Tjørve E, Tjørve KMC. A unified approach to the Richards-model family for use in growth analyses: why we need only two model forms. J Theor Biol 2010;267:417-425.

6. Fitzhugh HA. Analysis of growth curves and strategies for altering their shape. J Anim Sci 1976;42(4):1036-1051.

7. Abreu UG, Cobuci JA, da Silva MVGB, Sereno JRB. Uso de modelos no lineales para el ajuste de la curva de crecimiento de bovinos Pantaneiros. Arch Zootec 2004;53(204):367-370.

8. Noor RR, Saefuddin A, Talib C. Comparison on accuracy of Logistic, Gompertz and von Bertalanffy models in predicting growth of new born calf until first mating of Holstein Friesian heifers. J Ind Trop Anim Agric 2012;37(3):151-160.

9. Gbangboche AB, Glele-Kakai R, Salifou S, Albuquerque LGD, Leroy PL. Comparison of non-linear growth models to describe the growth curve in West African Dwarf sheep. Animal 2008;2(7):1003-1012.

10. Malhado CHM, Carneiro PLS, Affonso PRAM, Souza Jr AAO, Sarmento JLR. Growth curves in Dorper sheep crossed with the local Brazilian breeds, Morada Nova, Rabo Largo, and Santa Inês. Small Ruminant Res 2009;84(1):16-21. 
11. Behzadi MRB, Aslaminejad AA. A comparison of neural network and nonlinear regression predictions of sheep growth. J Anim Vet Adv 2010;9(16):2128-2131.

12. Perotto D, Cue RI, Lee AJ. Comparison of nonlinear functions for describing the growth curve of three genotypes of dairy cattle. Can J Anim Sci 1992;72(4):773-782.

13. Berry DP, Horan B, Dillon P. Comparison of growth curves of three strains of female dairy cattle. Anim Sci 2005;80(2):151-160.

14. McManus CM, Louvandini H, Campos VAL. Nonlinear growth curves for weight and height in four genetic groups of horses. Ciencia Anim Bras 2010;11(1):80-89.

15. Díaz HM. Indicaciones preliminares y el establecimiento de un módulo de producción de leche orgánica [tesis licenciatura]. Chapingo, Estado de México: Universidad Autónoma Chapingo; 2002.

16. Del Razo ROE. Características productivas y nivel de selenio y hormonas tiroideas en vacas lecheras suplementadas con bolos con selenio, bolos con selenio y yodo o bolos con yodo [tesis maestría]. Chapingo, Estado de México: Universidad Autónoma Chapingo; 2002.

17. Qing QBS. Comparison of four growth curve models in Angus cow: An application of Bayesian nonlinear mixed model. Report Presented to the Faculty of the Graduate School of the University of Texas at Austin; 2012.

18. SAS. SAS User's Guide: Statistics (version 9.3 ed.). Cary NC, USA: SAS Inst. Inc. 2016.

19. Craig BA, Schinckel AP. Nonlinear mixed effects model for swine growth. Prof Anim Scient 2001;17(4):256-260.

20. Schinckel AP, Li N, Preckel PV, Einstein ME, Miller D. Development of a stochastic pig compositional growth model. Prof Anim Scient 2003;19(3):255-260.

21. Strathe AB, Danfaer A, Sorensen H, Kebreab E. A multilevel nonlinear mixed-effects approach to model growth in pigs. J Anim Sci 2010;88(2):638-649.

22. Echeverri ZJ, Salazar RV, Parra SJ. Análisis comparativo de los grupos genéticos Holstein, Jersey y algunos de sus cruces en un hato lechero del Norte de Antioquia en Colombia. Zoot Trop 2011;29(1):49-59.

23. Posada OS, Rosero NR, Rodríguez N, Costa CA. Estimación de parámetros de curvas de crecimiento de ganado Nellore criado en confinamiento. Rev MVZ Córdoba 2011;16(3):2701-2710. 
24. Domínguez-Viveros J, Rodríguez-Almeida FA, Núñez-Domínguez R, Ramírez-Valverde R, Ortega-Gutiérrez A, Ruiz-Flores A. Ajuste de modelos no lineales y estimación de parámetros de crecimiento en bovinos Tropicarne. Agrociencia 2013;47(1):25-34.

25. Brown J, Fizugh H, Cartwright TC. A comparison of nonlinear models for describing weight-age relationships in cattle. J Anim Sci 1976;42(4):810-818.

26. Mazzini AR de A, Muniz JA, Silva FF, de Aquino LH. Curva de crescimento de novilhos Hereford: heterocedasticidade e resíduos autorregressivos. Cienc Rural 2005;35(2):422427.

27. Herrera RAC, Vergara GOD, Cerón MMF, Agudelo-Gómez D, Arboleda ZEM. Curvas de crecimiento en bovinos cruzados utilizando el modelo Brody. Livest Res Rural Dev 2008, http://www.lrrd.org/lrrd20/9/herr20140.htm. Consultado Dic 11, 2017.

28. Oliveira HN, Lôbo RB, Pereira CS. Comparação de modelos não-lineares para descrever o crescimento de fêmeas da raça Guzerá. Pesqui Agropecu Bras 2000;35(9):1843-1851.

29. Mgbere OO, Olutogun O. A Comparison of non-linear models for describing weight-age relationships in N'Dama cattle. J Appl Anim Res 2002;22(2):225-230.

30. Moreira RP, Mercadente MEZ, Pedrosa VB, Cyrillo JNSG, Henrique W. Growth curves of the Caracu breed. Ciências Agrárias 2016;37(4):2749-2758.

31. Gbangboche AB, Alkoiret TI, Toukourou Y, Kagbo A, Mensah GA. Growth curves for different body traits of Lagune Cattle. Res J Anim Sci 2011;5(2):17-24.

32. Vargas-Leitón B, Cuevas-Abrego M. Modelo estocástico para estimación de valores económicos de rasgos productivos y funcionales en bovinos lecheros. Agrociencia 2009;43(8):881-893.

33. López de Torre G, Candotti JJ, Reverter A, Bellido MM, Vasco P, García LJ, Brinks JS. Effects of growth curve parameters on cow efficiency. J Anim Sci 1992;70(9):2668-72. 